ARTICULOS ORIGINALES

Rev Chil Salud Pública 2020,

Vol 24(1): 11-22

\title{
ASOCIACIÓN ENTRE MARCADORES DE POSICIÓN SOCIAL Y ADHERENCIA AL TRATAMIENTO DE LA HIPERTENSIÓN ARTERIAL EN COLOMBIA
}

\author{
ASSOCIATION BETWEEN SOCIAL POSITION INDICATORS AND \\ HYPERTENSION TREATMENT ADHERENCE IN COLOMBIA
}

\section{RESUMEN}

Introducción: Generar evidencia sobre los efectos de la posición social en la adherencia terapéutica en personas con hipertensión arterial (HTA) en Colombia.

Materiales y Métodos: Se realizó un estudio transversal, a partir de mediciones cuantitativas del Programa de Determinantes Sociales e Inequidades en el Control de la HTA en Colombia. Se desarrollaron análisis descriptivos e inferenciales (regresión logística) para modelar las asociaciones.

Resultados: Se evidenció una alta proporción de adherencia, siendo mayor para cumplimiento farmacológico y las citas (>50\%). Las personas con menor educación e ingresos tienen menor posibilidad de adherirse al tratamiento farmacológico; mientras que quienes tienen mejor posición socioeconómica tienen menor posibilidad de adherirse a las citas y a las conductas saludables. Los afrocolombianos tienen menor posibilidad de adherirse al tratamiento farmacológico, a las citas y a la actividad física.

Discusión: Existe una brecha en el logro de la adherencia a tratamiento de HTA en Colombia, debido a condiciones socioeconómicas y étnica/raciales.

Palabras claves: Estatus económico, Etnia, Adherencia al tratamiento, Hipertensión

Silvia Patricia Quiroz Facultad de Ciencias de la Salud. Universidad Tecnológica del Chocó, Colombia

d-silvia.quiroz@utch.edu.co

Diana María Agudelo Facultad de Ciencias Sociales. Universidad de los Andes, Colombia

Diego Iván Lucumi Escuela de Gobierno Alberto Lleras Camargo.

Universidad de los Andes, Colombia

Graciela Mentz Escuela de Medicina, University of Michigan, Ann Arbor, USA

Recibido 14-01-2019 Aceptado 19-08-2019 arterial

\section{ABSTRACT}

Introduction: To generate evidence on the effects of social position on therapeutic adherence among individuals with hypertension (HT) in Colombia.

Materials and Methods: A cross-sectional study was carried out, using quantitative data from the Social Determinants and Inequities in the Control of HT Program in Colombia. Descriptive and inferential analyses (logistic regression) were developed to model the associations.

Results: The prevalence of adherence was high, especially for pharmacological treatment and compliance with appointments $(>50 \%)$. Individuals with less education and lower income are less likely to adhere to pharmacological treatment, while those with higher socioeconomic status are less likely to adhere to appointments and healthy behaviors. Afro-Colombians were less likely to adhere to pharmacological treatment, appointments, and indications regarding physical activity.

Discussion: There is a gap in HT treatment adherence in Colombia, due to socioeconomic and ethnic/racial conditions.

Key words: Economic status, Ethnicity, Treatment adherence, Arterial hypertension. 


\section{INTRODUCCIÓN}

La hipertensión arterial (HTA) constituye el principal factor de riesgo biológico de numerosos padecimientos crónicos y contribuye notablemente a la carga de mortalidad y discapacidad prematura en todos los países del mundo. Para reducir el riesgo asociado con la HTA, las personas diagnosticadas deben mantener controladas las cifras tensionales. Sin embargo, aunque se han promovido diferentes estrategias, el éxito ha sido bajo ${ }^{1}$. Estudios en diferentes países han mostrado proporciones de control de la HTA que oscilan entre $6.7 \%$ y $19.2 \%$, siendo mayores en países con más altos ingresos ${ }^{2-4}$. Por ejemplo, Colombia reportó menores proporciones de control de la HTA (15\%) en comparación con países vecinos con mayores ingresos como México $(19.2 \%)$ o Argentina $(18 \%)^{2}$.

Un requisito fundamental para el control de la HTA es que las personas sean diagnosticadas oportunamente e intervenidas adecuadamente mediante tratamientos farmacológicos (toma de medicamentos en la hora, frecuencia y dosis correcta) y no farmacológicos (alimentación saludable, actividad física y asistencia a las citas) ${ }^{1}$. Uno de los problemas más frecuentes en personas con HTA es la falta de adherencia a estos tratamientos ${ }^{1,5,6}$. La adherencia terapéutica puede ser definida como el grado en que el comportamiento de una persona de forma activa y voluntaria se corresponde con las recomendaciones acordadas con un prestador de asistencia sanitaria con el fin de lograr un resultado terapéutico desea$\mathrm{do}^{6-7}$.

Según la Organización Mundial de la Salud (2013), la baja adherencia terapéutica es más grave en condiciones crónicas como la HTA, y es aún más compleja en países de medianos y bajos ingresos económicos, representando un grave problema de salud pública con implicaciones tanto a nivel individual como colectivo. En regiones de Colombia, la evidencia ha sido contradictoria. Algunos estudios reportan altos porcentaje de adherencia a los tratamientos antihipertensivos, que oscilan entre el 88 y $98 \%{ }^{8-10}$, mientras que otros estudios reportan bajos porcentajes de adherencia $(9.4-51 \%)^{11-13}$. Por tanto, la evidencia sobre la adherencia terapéutica en la HTA en Colombia aún es insuficiente ${ }^{14}$.

Por otro lado, estudios principalmente en países de altos ingresos económicos como Estados Unidos, sugieren que la posición social, dada por la ubicación de la persona en la estructura social y medida en función de la posición socioeconómica (PSE), la condición étnica/racial y el género es "causa social fundamental" de los procesos de salud y enfermedad, incluida la adherencia terapéutica ${ }^{15-16}$.

En este orden, un estudio en España encontró una asociación inversamente proporcional entre nivel educativo y adherencia terapéutica medida a través del cumplimiento al tratamiento farmacológico en personas mayores de 60 años $^{17}$. Mientras que en Colombia se han reportado asociaciones positivas, en donde las personas con mayor PSE tienden a tener mejor adherencia terapéutica ${ }^{8,18,19}$.

A pesar de esta evidencia, una revisión sistemática reciente indica que el conocimiento actual sobre la asociación entre PSE y adherencia es extremadamente pobre, ya que, aunque los análisis combinados muestran que una mayor PSE se asocia con una disminución del $11 \%$ en el riesgo ajustado de la falta de adherencia, la heterogeneidad entre los estudios es muy alta y las medidas de PSE están incompletas y no se integran ${ }^{20}$.

Con relación a la etnia/raza la evidencia ha mostrado un patrón, que indica que quienes históricamente han tenido una mayor posición social dada la etnia/raza (blancos y mestizos) tienden a tener mayor adherencia terapéutica, por tanto tienen más posibilidades de control de la HTA ${ }^{21}$. Hasta la fecha, no se encontraron estudios sobre la asociación entre etnia/raza y adherencia terapéutica en Colombia, a diferencia de otros países, donde la evidencia alrededor del tema está más consolidada $22-24$.

Los efectos de la posición social sobre la adherencia pueden ser el resultado de tres mecanismos: i) la influencia de la posición social sobre la disponibilidad y accesibilidad a recursos, lo cual puede generar riesgos, exposiciones y vulnerabilidades en los individuos ${ }^{15-16}$; iii) los roles y comportamiento que se asumen en diferentes grupos sociales influenciados por las experiencias, creencias, percepciones y aptitudes ${ }^{25}$; y iii) el estrés relacionado con situaciones adversas derivadas de la posición social, que además actúa en doble vía, generando alteraciones fisiológicas o conllevando a realizar comportamientos de riesgo para la salud como fumar o hacer menos actividad física ${ }^{25}$.

La evidencia anterior da cuenta de potenciales inequidades en salud ${ }^{26}$. Esta evidencia plantea algunas debilidades importantes en intentos previos para comprender la asociación entre posición social y adherencia terapéutica, dejando como resultado que en muchos casos, como lo plantea la revisión 
sistemática de Alsabbagh et al. ${ }^{20}$, algunos indicadores de posición social no puedan considerarse un fuerte predictor de la adherencia debido a que la evidencia que respalda esta visión sigue siendo, en el mejor de los juicios, teórica, al menos con respecto a la adherencia farmacológica.

Bajo este contexto, el propósito de este estudio fue generar evidencia sobre la asociación entre posición social y adherencia farmacológica y no farmacológica en personas con HTA. Esta apuesta permitió valorar la adherencia terapéutica como un evento cuyo éxito no solo depende del sector sanitario, sino que también es necesaria una mirada más integral, lo que necesariamente implica un abordaje interdisciplinar, dado la diversidad de factores y mecanismos que inciden.

\section{MATERIALES Y MÉTODOS}

Tipo de estudio: Se realizó un estudio transversal, utilizando los datos de la primera medición cuantitativa en el año 2015 del Programa "De todo Corazón": Determinantes Sociales e Inequidades en el Control de la Hipertensión Arterial. Este programa tiene un enfoque de modelos mixtos de investigación siguiendo un diseño longitudinal, a través del cual se pretenden evaluar factores contextuales e individuales que inciden en la calidad de vida y la morbilidad de pacientes con HTA en Bogotá, MedeIlín y Quibdó. Como resultado de la investigación se pretende, además de identificar el impacto de las inequidades en salud sobre la HTA, establecer un modelo integral del problema que incluya variables que trasciendan el modelo médico tradicional y que puedan servir de insumo para orientar la implementación de la normatividad actual en Colombia.

Diseño muestral: El estudio usó dos tipos de muestreo. Primero, un muestreo de criterio o propósito de tipo heterogéneo para seleccionar las ciudades y las Entidades Administradoras de Planes de Beneficios - EAPB con programas de control de la HTA activos. Este tipo de muestreo es usado en investigación cualitativa, en donde existe una intención deliberada de examinar las variaciones o similitudes que pueda tener el fenómeno en diferentes contextos ${ }^{27}$. Las ciudades seleccionadas fueron Bogotá, Medellín y Quibdó, dadas sus diferencias en términos de niveles de pobreza, e infraestructura de servicios de salud (particularmente al comparar Quibdó con las otras dos ciudades).

Para la selección de las EAPB se garantizó que atendiera población afiliada al régimen subsidiado y contributivo en salud, tuvieran una cobertura igual o superior al tamaño de muestra de cada ciudad, contarán con un registro actualizado de las personas que forman parte del programa y tuvieran disposición de participar en el estudio.

La segunda etapa del muestreo implicó seleccionar personas usando un muestreo probabilístico estratificado basado en la distribución de edad y sexo, que permitió tomar una muestra 'representativa' de individuos dentro de cada institución, con el sesgo de selección mínimo.

Con el objetivo de aumentar el nivel de representatividad de la muestra y controlar aspectos como la no respuesta, se establecieron pesos muéstrales. Para este estudio en particular se analizaron los datos de 258 personas entre 45 y 70 años de edad, a quienes se les aplicó una encuesta en las instalaciones de las universidades vinculadas al proyecto en cada una de las ciudades seleccionadas, previo cumplimiento de los criterios de inclusión (personas mayores de 18 años, tener un diagnóstico de HTA, hacer parte del programa de HTA en la institución seleccionada y tener manejo médico ambulatorio). Además, se tuvo en cuenta como criterio de exclusión aquellas personas con alguna discapacidad cognitiva, neurológica, motora o psiquiátrica.

\section{Definición de variables e instrumentos}

Posición social: Este constructo se evaluó mediante dos grupos de variables: Posición socioeconómica (PSE) y etnia/raza. PSE: se midió mediante el nivel educativo más alto alcanzado (no estudio/primaria incompleta, primaria completa, diploma de bachillerato, grado estudios técnicos, grado de estudios tecnológicos, grado universitario y postgrado), el nivel de ingresos en función con el salario mínimo legal vigente en Colombia (SMLV) y la ocupación según la actividad que realizó en el último mes; Etnia/raza: se midió mediante dos variables obtenidas a partir del instrumento PERLA ${ }^{28}$. Este instrumento ha sido validado en países de Latino América. La primera variable se corresponde con la condición racial, y es definida en función con el criterio del entrevistador fundamentado en características fenotípicas. La segunda variable se corresponde con el auto reporte de la condición étnica. Para el propósito de este estudio, ambas variables se recodificaron así: indígena, negro/afrocolombiano y otros.

Adherencia terapéutica: se midió mediante variables obtenidas a partir del test de Morisky-Green-Le- 
vine (1986) y el cuestionario Martin-Bayarre-Grau (2008) ${ }^{29-30}$. Se valoraron dos componentes de la adherencia terapéutica. En primer lugar, el cumplimiento del tratamiento farmacológico y no farmacológico. El farmacológico se evaluó por medio del auto reporte de la toma de la medicación y el no farmacológico a partir de preguntas sobre el cumplimiento a las citas, la dieta baja en sal y la actividad física.

Consideraciones éticas: el estudio fue avalado por el comité de Ética de la Universidad de los Andes y de las instituciones participante, considerándose de riesgo mínimo y se acoge a la resolución 8430 de 1993 del Ministerio de Salud y Protección Social de Colombia.

Análisis estadístico: Se aplicaron análisis descriptivos para explorar los datos y determinar la distribución estadística de las medidas y los modelos más apropiados para el análisis propuesto. Se eliminaron los valores influyentes o atípicos (valores extremos no plausibles que pueden influir en las asociaciones).

Se estimaron correlaciones entre variables de posición social para evaluar la posible colinealidad y tomar decisiones sobre su inclusión en los modelos. Las variables evaluadas fueron: educación, ingresos, ocupación y etnia/raza (prueba de Chi - cuadrado). Posteriormente, se estimaron modelos bivariados mediante regresión logística, para analizar la asociación entre cada una de las variables de adherencia terapéutica y la posición social, y luego se estimaron modelos logísticos multivariados incluyendo las variables de forma conjunta y ajustando por sexo y edad.

Las categorías de referencias se asignaron a los grupos que según la evidencia están en mejor posición con referencia al evento estudiado. En el caso de la ocupación, se designó a los pensionados puesto que cumplen dos características que favorecen la adherencia, tales como acceso a recursos monetarios y mayor disposición de tiempo.

Los análisis se realizaron en el software Stata Versión 13 (College Station, TX: Stata Corp).

\section{RESULTADOS}

Se obtuvo una muestra final de 258 participantes de Bogotá (32.56\%), Medellín (37.21\%) y Quibdó $(30.23 \%)$ cuyo promedio de edad fue de 58 años $(\mathrm{DE}=6.59)$ y con una mayor proporción de mujeres $(62.40 \%)$.

La tabla 1 muestra que más del 50\% de los par- ticipantes tenía secundaria completa y/o estudios superiores, vive con menos de 2 salarios mínimos vigentes y tiene algún tipo de ocupación remunerada (empleado, independiente y pensionado). Con

Tabla 1. Descripción de las variables de posición social y adherencia.

\begin{tabular}{|c|c|c|}
\hline Variables & $\mathbf{n}$ & $\%$ \\
\hline \multicolumn{3}{|l|}{ Posición Socioeconómica - PSE } \\
\hline \multicolumn{3}{|l|}{ Educación } \\
\hline Menos de primaria & 48 & 18.6 \\
\hline Primaria completa & 60 & 23.2 \\
\hline Secundaria completa & 58 & 22.4 \\
\hline Más de secundaria & 92 & 35.7 \\
\hline \multicolumn{3}{|l|}{ Ingresos } \\
\hline Menos de un SMLV & 72 & 27.9 \\
\hline Entre 1 y 2 SMLV & 83 & 32.1 \\
\hline Entre 2 y 4 SMLV & 40 & 15.5 \\
\hline Más de 4 SMLV & 63 & 24.5 \\
\hline \multicolumn{3}{|l|}{ Ocupación } \\
\hline Empleado & 67 & 26.0 \\
\hline Independiente & 68 & 26.4 \\
\hline Desempleado & 16 & 6.2 \\
\hline Trabajo en el hogar & 55 & 21.4 \\
\hline Pensionado/Jubilado & 51 & 19.8 \\
\hline \multicolumn{3}{|l|}{ Etnia/Raza } \\
\hline Indígena & 8 & 3.3 \\
\hline Negro/mulato/afrocolombiano & 88 & 36.2 \\
\hline Blanco/Mestizo & 147 & 60.5 \\
\hline \multicolumn{3}{|l|}{ Variables de adherencia } \\
\hline \multicolumn{3}{|l|}{ Toma de medicamentos } \\
\hline Adherente & 132 & 52.2 \\
\hline No adherente & 121 & 47.2 \\
\hline \multicolumn{3}{|l|}{ Asistencia a citas } \\
\hline Adherentes & 206 & 81.1 \\
\hline No adherentes & 48 & 18.9 \\
\hline \multicolumn{3}{|l|}{ Consumo de sodio } \\
\hline Adherentes & 155 & 66.5 \\
\hline No adherentes & 78 & 33.5 \\
\hline \multicolumn{3}{|l|}{ Actividad física } \\
\hline Adherentes & 119 & 50.6 \\
\hline No adherentes & 116 & 49.3 \\
\hline
\end{tabular}

$\mathrm{n}=$ tamaño de la muestra

$\%=$ Porcentaje 
relación a la etnia/raza la mayor proporción de los participantes se identificaron con la categoría de blanco o mestizo (60.49\%), y se evidenció una baja representatividad de la población indígena (3.29\%), razón por la cual esta categoría no fue incluida en los análisis posteriores.

Adicionalmente, en la tabla 1 se muestra una descripción de las variables de adherencia utilizadas en este estudio, en donde se evidencia que la mayoría de los encuestados refieren adherirse o seguir las recomendaciones con respecto a la toma de medicamentos, asistencias a citas, consumo de sodio y actividad física; presentando una mayor proporción en la variable de asistencia a citas (81.1\%).
En la tabla 2 se observan los resultados relacionados con la asociación entre adherencia y posición social mostrando que las personas con mayor posición social tienen mayor adherencia farmacológica, de manera que quienes tienen un nivel educativo igual o menor a la primaria completa, ganan menos de 3 SMLV o quienes laboran de forma independiente tienen menos probabilidades de adherencia farmacológica, en comparación con las personas de mayor posición según cada categoría. Asimismo, las personas afrodescendientes tienen menos posibilidades de adherirse al tratamiento farmacológico. Sin embargo, estos hallazgos no alcanzaron significancia estadística en ninguno de los modelos empleados.

Tabla 2 . Asociación entre adherencia farmacológica y posición social

\begin{tabular}{|c|c|c|c|c|}
\hline \multirow[t]{2}{*}{ Variables } & \multicolumn{2}{|c|}{ Simple } & \multicolumn{2}{|c|}{ Ajustado } \\
\hline & OR (ES) & IC & OR & IC \\
\hline \multicolumn{5}{|l|}{ Educación } \\
\hline Menos de primaria & $0.65(0.37)$ & $0.21-2.03$ & $0.47(0.34)$ & $0.11-1.95$ \\
\hline Primaria completa & $0.90(0.50)$ & $0.29-2.71$ & $0.79(0.50)$ & $0.23-2.70$ \\
\hline Secundaria & $1.19(0.72)$ & $0.36-3.89$ & $1.25(0.66)$ & $0.44-3.52$ \\
\hline Estudios superiores & \multicolumn{4}{|c|}{ Referencia } \\
\hline \multicolumn{5}{|l|}{ Ingresos } \\
\hline Menos de 1 SMLV & $1.24(0.75)$ & $0.38-4.08$ & $0.80(0.50)$ & $0.23-2.70$ \\
\hline Entre 1 y 2 SMLV & $0.89(0.50)$ & $0.29-2.71$ & $0.69(0.47)$ & $0.18-2.60$ \\
\hline Entre 3 y 4 SMLV & $2.12(1.55)$ & $0.50-8.89$ & $1.57(1.06)$ & $0.41-5.93$ \\
\hline Más de 4 SLMV & \multicolumn{4}{|c|}{ Referencia } \\
\hline \multicolumn{5}{|l|}{ Ocupación } \\
\hline Empleado & $1.61(0.95)$ & $0.51-5.10$ & $1.31(0.92)$ & $0.32-5.23$ \\
\hline Independiente & $0.78(0.44)$ & $0.25-2.39$ & $0.91(0.63)$ & $0.23-3.57$ \\
\hline Trabajo en el hogar & $1.32(0.72)$ & $0.45-3.84$ & $1.54(1.36)$ & $0.27-8.67$ \\
\hline Desempleado & $1.62(1.42)$ & $0.29-9.06$ & $1.99(2.13)$ & $0.24-16.17)$ \\
\hline Pensionado & \multicolumn{4}{|c|}{ Referencia } \\
\hline \multicolumn{5}{|l|}{ Etnia/raza } \\
\hline Negros/ afrocolombianos & $0.55(0.27)$ & $0.20-1.46$ & $1.38(1.06)$ & $0.30-6.27$ \\
\hline Blancos/Mestizos & \multicolumn{4}{|c|}{ Referencia } \\
\hline
\end{tabular}

Análisis ajustados por edad y sexo

OR=coeficiente de regresión logística -razones de momios

IC: Intervalos de Confianza 95\%

$\mathrm{p}=$ valor de $\mathrm{p}^{*}<0.05 \quad-* *<0.01$ 
En la tabla 3 se muestran los resultados de la asociación entre posición social y adherencia en términos de la asistencia a las citas médicas. Se encontró que las personas con primaria o menos y quienes tiene menos de 4 SMLV tienen más posibilidades de cumplir con las citas de control en comparación con quienes tienen estudios superiores y tienen mejores ingresos. Los empleados y trabajadores independientes tienen menos posibilidades de adherirse a las citas, mientras que las personas trabajadoras del hogar tienen más posibilidad de cumplir las citas en comparación con los pensionados.

Por otra parte, las personas negras/afrodescendientes tienen menos posibilidades de adherirse a las citas de control, pero el efecto cambia en el modelo ajustado. Sin embargo, estos hallazgos carecen de significancia estadística.

Tabla 3. Asociación entre adherencia a citas y posición social

\begin{tabular}{|c|c|c|c|c|}
\hline \multirow[t]{2}{*}{ Variables } & \multicolumn{2}{|c|}{ Simple } & \multicolumn{2}{|c|}{ Ajustado } \\
\hline & OR & IC & OR & IC \\
\hline \multicolumn{5}{|l|}{ Educación } \\
\hline Menos de primaria & 3.63 & $0.12-18.12$ & 0.70 & $0.11-4.33$ \\
\hline Primaria completa & 3.45 & $0.93-12.73$ & 2.76 & $0.50-15.23$ \\
\hline Secundaria & 0.74 & $0.23-2.29$ & 0.53 & $0.13-2.21$ \\
\hline Estudios superiores & \multicolumn{4}{|c|}{ Referencia } \\
\hline \multicolumn{5}{|l|}{ Ingresos } \\
\hline Menos de 1 SMLV & 2.11 & $0.54-8.12$ & 1.04 & $0.13-7.87$ \\
\hline Entre 1 y 2 SMLV & 1.29 & $0.40-4.17$ & 2.61 & $0.38-17.88$ \\
\hline Entre 3 y 4 SMLV & 1.21 & $0.31-4.78$ & 1.78 & $0.38-8.36$ \\
\hline Más de 4 SLMV & \multicolumn{4}{|c|}{ Referencia } \\
\hline \multicolumn{5}{|l|}{ Ocupación } \\
\hline Empleado & 0.53 & $0.12-2.21$ & 0.22 & $0.03-1.45$ \\
\hline Independiente & 0.64 & $0.15-2.61$ & 0.86 & $0.13-5.65$ \\
\hline Trabajo en el hogar & 8.48 & $0.84-85.51$ & 9.76 & $0.49-19.00$ \\
\hline Pensionado & \multicolumn{4}{|c|}{ Referencia } \\
\hline \multicolumn{5}{|l|}{ Etnia/raza } \\
\hline Negros/ afrocolombianos & 0.51 & $0.20-1.25$ & 1.13 & $0.14-8.64$ \\
\hline Blancos/Mestizos & \multicolumn{4}{|c|}{ Referencia } \\
\hline
\end{tabular}

Análisis ajustados por edad y sexo

$\mathrm{OR}=$ coeficiente de regresión logística -razones de momios

IC: Intervalos de Confianza 95\%

$\mathrm{p}=$ valor de $\mathrm{p}^{*}<0.05 \quad-* *<0.01$ 
La tabla 4 muestra los resultados de asociación entre adherencia y actividad física indicando que las personas que tienen primaria o menos y las de menores ingresos tienen mayor probabilidad de adherirse a la actividad fisica, en comparación con quienes tienen estudios superiores o mayores ingresos, respectivamente. Con relación a la ocupación, se encontró que las personas que laboran como empleados, independientes y trabajadores del hogar tienen menos posibilidades de adherise a la actividad fisica en comparación con los pensionados. Por ultimo, los negros/afrodescendientes tienen menos posibilidades de adherise a la actividad fisica, lo cual cambia de efecto en el modelo ajustado, en donde estos tiene más posibilidades de adherirse a la actividad fisica. Estos hallazgos pierden significancia estadistica en el modelo ajustado.

Tabla 4 . Asociación entre actividad fisica y posición social.

\begin{tabular}{|c|c|c|c|c|}
\hline \multirow[t]{2}{*}{ Variables } & \multicolumn{2}{|c|}{ Simple } & \multicolumn{2}{|c|}{ Ajustado } \\
\hline & OR & IC & OR & IC \\
\hline \multicolumn{5}{|l|}{ Educación } \\
\hline Menos de primaria & $3.39 *$ & $1.00-11.53$ & 1.49 & $0.32-6.87$ \\
\hline Primaria completa & 2.35 & $0.75-7.37$ & 1.56 & $0.46-5.30$ \\
\hline Secundaria & 0.56 & $0.16-1.96$ & 0.63 & $0.19-2.05$ \\
\hline Estudios superiores & \multicolumn{4}{|c|}{ Referencia } \\
\hline \multicolumn{5}{|l|}{ Ingresos } \\
\hline Menos de 1 SMLV & 2.32 & $0.68-7.87$ & 1.46 & $0.40-5.35$ \\
\hline Entre 1 y 2 SMLV & 2.11 & $0.70-6.35$ & 1.68 & $0.51-5.57$ \\
\hline Entre 3 y 4 SMLV & 1.52 & $0.28-8.12$ & 1.66 & $0.33-8.36$ \\
\hline Más de 4 SLMV & \multicolumn{4}{|c|}{ Referencia } \\
\hline \multicolumn{5}{|l|}{ Ocupación } \\
\hline Empleado & $0.25^{*}$ & $0.06-0.99$ & 0.43 & $0.10-1.82$ \\
\hline Independiente & $0.18^{* *}$ & $0.05-0.61$ & 0.34 & $0.08-1.34$ \\
\hline Trabajo en el hogar & 0.53 & $0.17-1.62$ & 0.75 & $0.12-4.63$ \\
\hline Pensionado & \multicolumn{4}{|c|}{ Referencia } \\
\hline \multicolumn{5}{|l|}{ Etnia/raza } \\
\hline Negros/ afrocolombianos & 0.72 & $0.25-2.06$ & 1.69 & $0.26-10.72$ \\
\hline Blancos/Mestizos & \multicolumn{4}{|c|}{ Referencia } \\
\hline
\end{tabular}

Análisis ajustados por edad y sexo

$\mathrm{OR}=$ coeficiente de regresión logística -razones de momios

IC: Intervalos de Confianza 95\%

$\mathrm{p}=$ valor de $\mathrm{p}^{*}<0.05 \quad-* *<0.01$ 
En la tabla 5 se muestran los resultados de la asociación entre consumo de sodio y posición social. Los datos indican que las personas con menores niveles educativos y menores ingresos tienen más posibilidades de adherirse a la dieta baja en sodio. Con relación, a la ocupación se encontró que los empleados y trabajadores independientes tienen menos posibilidades de adherirse a la dieta baja en sodio, mientras que los trabajadores del hogar tienen más posibilidades de adherirse a la misma, siendo este último hallazgo estadisticamente significativo en el modelo ajustado. Finalmente, se muestra que las personas negras o afrodescendientes tienen más posibilidades de adherirse a la dieta baja en sodio, lo cual no es estadisticamente significativo en ninguno de los modelos.

Tabla 5. Asociación entre consumo de sodio y posición social

\begin{tabular}{|c|c|c|c|c|}
\hline \multirow[t]{2}{*}{ Variables } & \multicolumn{2}{|c|}{ Simple } & \multicolumn{2}{|c|}{ Ajustado } \\
\hline & OR & IC & OR & IC \\
\hline \multicolumn{5}{|l|}{ Educación } \\
\hline Menos de primaria & 3.07 & $0.88-10.66$ & 1.77 & $0.31-9.89$ \\
\hline Primaria completa & $4.05^{*}$ & $1.28-12.81$ & 1.45 & $0.43-4.83$ \\
\hline Secundaria & 3.23 & $0.92-11.29$ & 1.79 & $0.49-6.48$ \\
\hline Estudios superiores & \multicolumn{4}{|c|}{ Referencia } \\
\hline \multicolumn{5}{|l|}{ Ingresos } \\
\hline Menos de 1 SMLV & 2.51 & $0.66-9.49$ & 4.43 & $0.75-26.11$ \\
\hline Entre 1 y 2 SMLV & 1.75 & $0.48-6.37$ & $4.90^{*}$ & $1.20-20.02$ \\
\hline Entre 3 y 4 SMLV & 0.52 & $0.09-2.83$ & 1.81 & $0.35-9.24$ \\
\hline Más de 4 SLMV & \multicolumn{4}{|c|}{ Referencia } \\
\hline \multicolumn{5}{|l|}{ Ocupación } \\
\hline Empleado & 0.59 & $0.16-2.18$ & 1.11 & $0.22-5.55$ \\
\hline Independiente & 0.88 & $0.22-3.50$ & 1.96 & $0.40-9.50$ \\
\hline Trabajo en el hogar & $3.91^{*}$ & $1.11-13.80$ & $9.75^{*}$ & $1.11-85.26$ \\
\hline Pensionado & \multicolumn{4}{|c|}{ Referencia } \\
\hline Negros/ afrocolombianos & 1.13 & $0.43-2.91$ & 10.11 & $0.77-132$ \\
\hline Blancos/Mestizos & \multicolumn{4}{|c|}{ Referencia } \\
\hline
\end{tabular}

Análisis ajustados por edad y sexo

$\mathrm{OR}=$ coeficiente de regresión logística -razones de momios

IC: Intervalos de Confianza 95\%

$\mathrm{p}=$ valor de $\mathrm{p}^{*}<0.05 \quad-* *<0.01$ 


\section{DISCUSIÓN}

Los hallazgos más notables de este estudio se concentran en tres aspectos: i) se evidenció una alta proporción de adherencia, siendo mayor el cumplimiento al tratamiento farmacológico y a las citas en comparación con la adherencia a la actividad física y el consumo de sodio recomendado; ii) las personas con menor nivel educativo e ingresos tienen menor posibilidad de adherirse al tratamiento farmacológico; mientras que quienes tienen mayor educación e ingresos y los empleados e independientes tienen menor posibilidad de adherirse a la citas y las conductas saludables y iii) los afrodescendiente tienen menor posibilidad de adherirse al tratamiento farmacológico, a las citas y a la actividad física, sin embargo, la dirección del efecto cambia al ajustarse por variables de PSE.

El alto grado de adherencia reportado en este estudio es un hallazgo deseable, en tanto la evidencia ha demostrado que la adherencia es una de las estrategias conductuales que mejor permiten el control de la HTA ${ }^{1,6,7}$; y además es una de las acciones de prevención secundaria más eficientes para aumentar la supervivencia de los pacientes ${ }^{31}$.

Es importante destacar que el alto grado de adherencia reportado en este estudio puede vincularse a un sesgo de participación. Las personas que toman la decisión de participar en investigaciones en salud tienden a estar más informadas sobre su enfermedad, más motivadas y comprometidas con su manejo y asumen más comportamientos saludables en comparación con las que no participan ${ }^{32}$.

La discrepancia entre la adherencia farmacológica y las conductas saludables reportada en el primer hallazgo de este estudio, coinciden con evidencia reportada a nivel mundial, que muestran que las personas con HTA cumplen en mayor proporción con las recomendaciones farmacológicas y las citas en comparación con las no farmacológicas $7,14,33$. En Colombia, un estudio encontró que solo el $9.4 \%$ de los pacientes fueron adherentes a las recomendaciones no farmacológicas, y al igual que en este estudio encontraron que la adherencia a la actividad física fue la categoría con menor proporción de cumplimiento $(25 \%)^{34}$.

En la literatura reciente se han publicado diversos estudios sobre factores que intentan explicar el hallazgo anterior. A manera de resumen, este hallazgo se ha vinculado con la subvaloración y desconocimiento de los efectos protectores de la adherencia no farmacológica, el requerimiento de mayores recursos psicosociales y económicos para adherirse a conductas saludables y la presencia de barreras estructurales ${ }^{35,36}$.

Con respecto al segundo hallazgo notable de este estudio, en la literatura se ha discutido el efecto de la PSE sobre la adherencia farmacológica y no farmacológica, en contraste con los resultados aquí reportados, la evidencia contradice en algunos casos y reafirma en otros. Es clave mencionar que un punto de encuentro entre la literatura revisada y los haIlazgos de este estudio es que independientemente de la dirección o magnitud del efecto, la PSE tiene un impacto sobre la adherencia terapéutica, el cual varía en función de la conceptualización y el tipo de medidas utilizados para definir tanto los indicadores de PSE como la adherencia en sí misma.

Los estudios de Arrivillaga ${ }^{18}$ y Benavides et al ${ }^{19}$ muestran resultados similares con relación a la dirección de las asociaciones reportadas. Estos autores indican que las personas con menor PSE tienden a tener menor adherencia farmacológica, asociado al hecho de que por ejemplo un mayor nivel de educación confiere conocimientos, credenciales y redes sociales, que puede aumentar el entendimiento de las recomendaciones orales y escritas sobre el tratamiento e incrementar la posibilidad de que se adhieran a este. Por otra parte, el ingreso ofrece acceso a ciertas condiciones de vida que promueven el cuidado de la salud.

Se evidenció también que las personas con mayor nivel educativo, mayores ingresos y las empleadas e independientes tienen más riesgo de no ser adherentes a las conductas saludables. Esto puede asociarse al hecho de que las personas con mayor PSE pueden tener menos tiempo disponible para ejecutar ciertas conductas saludables, aunque puedan tener otro tipo de recursos. Un estudio en Cuba mostró resultados similares, indicando que los pacientes que tienen una vida laboral activa tenían menores posibilidades de adherirse. Esto fue relacionado con la presencia de diversas responsabilidades y obligaciones de los pacientes en el trabajo, lo cual provocó falta de autocuidado ${ }^{37}$.

Finalmente, el tercer hallazgo relevante de este estudio nos muestra que los afrodescendientes tienen menor posibilidad de adherirse al tratamiento de la HTA, lo cual es coherente con lo planteado en otros estudios. En Estados Unidos una investigación mostró que los afroamericanos tienen menor adherencia a la dieta saludable y a la actividad física en compa- 
ración con otros grupos étnicos ${ }^{38}$.

Los patrones culturales son aspectos claves a tener en cuenta en la explicación del hallazgo anterior, es decir, algunos grupos étnicos pueden consciente $\mathrm{o}$ inconscientemente participar de actividades para preservar las tradiciones y mantener la identidad de grupo, lo cual puede impactar sobre su propia salud ${ }^{39}$. Sin embargo, hay que considerar que, si bien la explicación cultural es factible, a su vez puede ser riesgosa si se desconocen la incidencia de factores estructurales; que, por un lado, pueden influenciar la cultura, contribuyendo en la definición de ciertos patrones de comportamiento en grupos étnicos específicos. Por otro lado, por sí mismos han jugado un papel determinante sobre el estado de salud de las minorías étnicas en Colombia. En un estudio cualitativo llevado a cabo en la ciudad de Quibdó, una región con alta concentración de afrocolombianos en Colombia ratifica lo descrito, dado que muestran que factores estructurales como el desempleo o la inseguridad pueden determinar el riesgo de HTA y generar disparidades en salud ${ }^{40}$.

Este estudio tuvo varias limitaciones que deben ser tenidas en cuenta al momento de valorar los resultados: i) el tipo de diseño transversal y no experimental no permite asumir causalidad ${ }^{41}$; ii) la medición indirecta de la adherencia mediante el uso de cuestionarios de auto reporte puede generar posibles sesgos de información ${ }^{42}$; y iii) Un posible sesgo de selección y participación pudo influir los resultados ${ }^{41}$, es decir, quienes aceptaron participar y acudieron a la entrevista pueden tener ciertas características comunes. Por ejemplo, ser quienes más cumplan las citas programadas, $y$, por ende, ser los más adherentes y controlados.

Los hallazgos reportados en este estudio ponen de relieve el papel interactivo de la posición social en el logro de la adherencia terapéutica en Colombia. A manera de conclusión se precisan dos aspectos. En primer lugar, estos hallazgos indican que existe una brecha en el logro de la adherencia al tratamiento de la HTA en Colombia, a razón de condiciones socioeconómicas y étnicas.

En segundo lugar, la discrepancia en términos de adherencia farmacológica y no farmacológica hace una invitación a reflexionar sobre la importancia que se le da a cada uno de estos aspectos, en términos de beneficios por parte de los pacientes y sus familias, los prestadores de servicios de salud y los tomadores de decisiones e implementadores de políticas para el manejo y control de la HTA.
Futuros estudios deben orientarse a explorar la interacción de mecanismos que influyen en la asociación entre posición social y adherencia terapéutica. Tal es el caso del estrés, que ha sido reconocido como un mecanismo que explica los vínculos entre algunos asuntos en salud y condiciones sociales ${ }^{25}$. De igual manera, se sugiere indagar por la influencia del género como variable de posición social, y de la implicación personal y el autocuidado como componentes claves en la medición de la adherencia terapéutica.

\section{REFERENCIAS BIBLIOGRÁFICAS}

1. Organización Mundial de la Salud. Información general sobre la hipertensión en el mundo: una enfermedad que mata en silencio, una crisis de salud pública [en línea]. 2013. [place unknown]. Disponible en: www. who.int/about/licensing/copyright_form/en/index.html

2. Sánchez R, Ayala M, Baglivo H, Velázquez C, Burlando G, Kohlmann O, et al. Guías Latinoamericanas de Hipertensión Arterial. Rev Chil Cardiol 2010;29:117144

3. Chow CK, Teo KK, Rangarajan S, Islam S, Gupta R, Avezum A, et al. Prevalence, awareness, treatment, and control of hypertension in rural and urban communities in high-, middle-, and low-income countries. JAMA 2013;310:959-68.

4. Adler AJ, Prabhakaran D, Bovet P, Kazi DS, Mancia G, Mungal-Singh $\mathrm{V}$, et al. Reducing cardiovascular mortality through prevention and management of raised blood pressure: a World Heart Federation Road Map. Glob Heart. 2015;10:111-22.

5. Martínez JW, Perea JAV, Jaramillo J, Betancur AMQ, Calderón V. Validación del cuestionario de adherencia al tratamiento anti hipertensivo Martín Bayarré Grau. Revista Médica de Risaralda. 2011;17(2).

6. Ortiz E. Psicología de la salud: una clave para comprender el fenómeno de la adherencia terapéutica. Rev Méd Chile. 2007;135(5):647-652.

7. Johnson S, Carlson D. Medical regimen adherence: concepts, assessment and interventions. Handbook of clinical health psychology. USA. 2014

8. Contreras S. Factores que influyen en la adherencia a tratamientos farmacológicos y no farmacológicos en los pacientes inscritos al programa de control de la hipertensión de una unidad básica de atención de Coomeva de Sincelejo en 2006. Revista de Salud Uni- 
norte. 2010;6(2):202-211

9. Isaza C, Moncada J, Mesa G, Osorio F. Efectividad del tratamiento hipertensivo en na muestra de pacientes colombianos. Biomedica. 2004;24(3):273-81.

10. Torres IEF. Adherencia a tratamientos en pacientes con factores de riesgo cardiovascular. Av Enferm. $2009 ; 27(2): 25-32$.

11. Casas M, Chavarro C, Cardona D. Adhesión al tratamiento antihipertensivo en dos municipios de Colombia 2010-2011. Hacia la promoción de la salud. 2013;8(1):81-96.

12. Castaño J, Echeverry C, Giraldo J, Maldonado A, Melo J, Maza G, et al. Adherencia al tratamiento de pacientes hipertensos atendidos en ASSbasalud ESE, Manizales (Colombia) 2011. Rev Fac Med. 2012;60(3):179197.

13. Herrera E. Adherencia al tratamiento en personas con hipertensión. Av Enferm. 2012;2(2):67-65.

14. Varela M. El reto de evaluar la adherencia al tratamiento de la hipertensión. Pensamiento psicológico. 2010;7(14):127-140.

15. Link BG, Phelan J. Social conditions as fundamental causes of disease. J Health Soc Behav. 1995:80-84.

16. Link BG, Phelan J. Social conditions as fundamental causes of health inequalities. Handbook of Medical sociology. 2010;6;3-17. 2010.

17. Tuesca-Molina R, Guallar-Castillón P, Banegas-Banegas JR, Graciani-Pérez Regadera A. Determinantes del cumplimiento terapéutico en personas mayores de 60 años en España. Gac Sanit. 2006;20(3):220-227.

18. Arrivillaga M, Ross M, Useche B, Alzate M, Correa1 D. Social position, gender role, and treatment adherence among Colombian women living with HIV/AIDS: social determinants of health approach. Rev Pan Salud Pública. 2009;26(6):502-10.

19. Benavides V, Jaramillo L, Rendón S, Valenzuela A, Pérez E, Mafla A. Determinantes de adherencia al tratamiento antihipertensivo de adultos $\geq 35$ años de edad. Univ Salud. 2013;15(2): 123-135.

20. Alsabbagh MH, Lemstra M, Eurich D, Lix LM, Wilson TW, Watson E, et al. Socioeconomic status and nonadherence to antihypertensive drugs: a systematic review and meta-analysis. Value Health. 2014;17(2):288 296.

21. Bosworth, H. Racial differences in blood pressu- re control: potential explanatory factors. Am J Med. 119(1):70.e9-15.

22. Francis CK. Hypertension, cardiac disease, and compliance in minority patients. Am J Med. 1991;91(1):S29-S36.

23. Holmes HM, Luo R, Hanlon JT, Elting LS, Suarez-Almazor M, Goodwin JS. Ethnic disparities in adherence to antihypertensive medications of medicare part D beneficiaries. J Am Geriatr Soc. 2012; 60(7):1298-30.

24. Traylor AH, Schmittdiel JA, Uratsu, CS, Mangione CM, Subramanian $U$. Adherence to cardiovascular disease medications: does patient-provider race/ethnicity and language concordance matter?. J Gen Inter Medicine. 2010; 25(11):1172-7.

25. Rugulies R, Aust, B, Sime L. Epidemiology of health and illness: a socio-psycho-physiological perspective. En: Sutton S, Baum A, Johnston M. (Eds.). London: SAGE; 2010. p. 42-68.

26. Braveman PA, Kumanyika S, Fielding J, LaVeist T, Borrell LN, Manderscheid R, et al. Health disparities and health equity: the issue is justice. Am J Public Health. 2011;101(S1): S149-S155.

27. Patton MQ. Enhancing the quality and credibility of qualitative analysis. Health Serv Res. 1999;34(5 Pt 2):1189-1208.

28. Telles E; Project on Race and Ethnicity in Latin America (PERLA). Pigmentocracies: ethnicity, race and color in Latin America. Chapel Hill: University of North Carolina Press; 2014.

29. 29. Morisky D, Green L, Levine D. Concurrent and predictive validity of a self-reported measure of medication adherence. Med Care. 1986;24:67-74.

30. Martin-Alfonso L, Bayarre-Vea HD, Grau-Abalo JA. Validación del cuestionario MBG (Martin-Bayarre-Grau) para evaluar la adherencia terapéutica en hipertensión arterial. Rev Cubana Salud Pública. 2008;34:7.

31. Orozco-Beltrán D, Carratalá-Munuera C, Gil-Guillén V. Mejorar la adherencia: una de las acciones más eficientes para aumentar la supervivencia de los pacientes en prevención secundaria. Rev Esp Cardiol Supl. 2015;15(E):12-18.

32. Tolonen H, Dobson A, Kulathinal S. Effect on trend estimates of the difference between survey respondents and non-respondents: results from 27 populations in the WHO MONICA Project. Eur J Epidemiol. 2005;20(11):887-898. 
33. Jankowska-Polanska B, Blicharska K, Uchmanowicz I, Morisky DE. The influence of illness acceptance on the adherence to pharmacological and non-pharmacological therapy in patients with hypertension. Eur J Cardiovasc Nur. 2016;15(7):559-568.

34. Lopez R, Mérida R, Varela A, María T, Rincón H, Velasco $P$, et al. Prevalencia y factores asociados a la adherencia al tratamiento no farmacológico en pacientes con hipertensión y diabetes en servicios de baja complejidad. Rev Fac Nac Salud Pública. 2015;33(2):192-199.

35. Ramos Morales LE. La adherencia al tratamiento en las enfermedades crónicas. Rev Cubana Angiol Cir Vasc. 2015;16(2):175-189.

36. Egan BM, Lackland DT and Cutler NE. Awareness, knowledge, and attitudes of older Americans about high blood pressure: implications for health care policy, education and research. Arch Intern Med. 2003;163:681-687.

37. Corugedo Rodríguez, MC, Libertad Martín A, Bayarre Vea H. Adherencia terapéutica en pacientes con hipertensión arterial del Policlínico Universitario. "Manuel Fajardo" en el Municipio Cruces, Cienfuegos, 2009. Rev Cubana Med Gen Integr. 2011;27(4):504-512.
38. Newton Jr RL, Griffith DM, Kearney WB, Bennett GG. A systematic review of weight loss, physical activity and dietary interventions involving African American men. Obes Rev. 2014 Oct;15 Suppl 4:93-106.

39. James DC. Factors influencing food choices, dietary intake, and nutrition-related attitudes among African Americans: application of a culturally sensitive model. Ethn Health. 2004 Nov;9(4):349-67.

40. Lucumi DI, Schulz AJ, Israel BA. Local Actors' Frames of the Role of Living Conditions in Shaping Hypertension Risk and Disparities in a Colombian Municipality. J Urban Health. 2016;93(2):345-363.

41. Hernández B, Velasco-Mondragón HE. Encuestas transversales. Salud Pública Méx. 2000; 42:447-455.

42. Rodríguez-López MR, Varela MT, Rincón-Hoyos, Velasco MM, Caicedo DM, Méndez F, et al. Prevalencia y factores asociados a la adherencia al tratamiento no farmacológico en pacientes con hipertensión y diabetes en servicios de baja complejidad. Rev Fac Nac Salud Pública. 2015;33(2):192-199. 